Classification

Physics Abstracts

42.65C

\title{
DIFFUSION STIMULÉE INDUITE PAR UNE ABSORPTION A DEUX PHOTONS
}

\author{
P. BOISSEL, G. HAUCHECORNE, F. KERHERVE et G. MAYER \\ Laboratoire d'Optique Quantique, Université Paris VI, Tour 13, \\ 4, place Jussieu, 75230 Paris Cedex 05, France
}

(Reçu le 16 juin 1978, révisé le 10 juillet 1978, accepté le 3 août 1978)

\begin{abstract}
Résumé. - Une absorption à deux photons, tout comme une absorption linéaire peut induire une diffusion du type Rayleigh thermique stimulé. Nous avons mis ce phénomène en évidence dans le benzène et le chloronaphtalène. Nous déduisons pour ces deux molécules une valeur de la section efficace de $4 \times 10^{-50} \mathrm{~cm}^{4}$.s et $10^{-48} \mathrm{~cm}^{4}$.s à un niveau d'énergie de $7,2 \mathrm{eV}$.
\end{abstract}

Abstract. - Two photon absorption, as well as linear absorption, can induce stimulated thermal Rayleigh scattering. We have demonstrated this phenomena in benzene and chloronaphtalene. The cross section for two photon absorption of these two molecules are respectively $4 \times 10^{-50} \mathrm{~cm}^{4} . \mathrm{s}$ and $10^{-48} \mathrm{~cm}^{4} . \mathrm{s}$ at an energy level of $7.2 \mathrm{eV}$.

La diffusion Rayleigh thermique stimulé par les milieux absorbants a été très étudiée vers les années 1970 (voir par exemple [1, 2, 3] ou [4] pour un article de revue). Mais toutes ces études se sont limitées au cas où le phénomène est induit par une absorption linéaire ou par un effet Raman [5]. Il nous a paru intéressant de mettre en évidence cette diffusion stimulée dans le cas d'une absorption à deux photons.

Mettons en présence deux ondes optiques

$$
E_{\mathrm{L}} \cos \left(\omega_{\mathrm{L}} t-k_{\mathrm{L}} z\right) \text { et } E_{\mathrm{S}} \cos \left(\omega_{\mathrm{S}} t+k_{\mathrm{S}} z\right)
$$

se propageant en sens inverse dans un milieu caractérisé par un indice d'absorption non linéaire $b$ défini par

$$
\frac{\mathrm{d} I}{\mathrm{~d} z}=-b I^{2}
$$

L'énergie cédée par ces deux ondes au milieu contiendra un terme oscillant à la fréquence $\omega_{\mathrm{L}}-\omega_{\mathrm{S}}$ de la forme :

$$
\begin{array}{r}
\dot{w}=2 b\left(I_{\mathrm{L}}+I_{\mathrm{S}}\right) \frac{c}{8 \pi} E_{\mathrm{L}} E_{\mathrm{S}} \cos \left\{\left(\omega_{\mathrm{L}}-\omega_{\mathrm{S}}\right) t-\right. \\
\left.-\left(k_{\mathrm{L}}+k_{\mathrm{S}}\right) z\right\}
\end{array}
$$

analogue à celui que donnerait une atténuation linéaire d'indice $\alpha$

$\dot{w}=\alpha \frac{c}{8 \pi} E_{\mathrm{L}} E_{\mathrm{S}} \cos \left\{\left(\omega_{\mathrm{L}}-\omega_{\mathrm{S}}\right) t-\left(k_{\mathrm{L}}+k_{\mathrm{S}}\right) z\right\}$.
Un raisonnement classique conduit donc à la conclusion que l'onde $\omega_{\mathrm{S}}$ sera amplifiée en présence de $\omega_{\mathrm{L}}$ avec un gain maximum en régime permanent

$$
g_{\mathrm{R}} I_{\mathrm{L}}=\alpha_{\mathrm{eq}} \frac{\omega_{\mathrm{S}} \beta_{T}}{c n C_{p}}\left(\frac{\partial \varepsilon}{\partial \rho}\right)_{T} \tau_{\mathrm{R}} I_{\mathrm{L}}=\Gamma \alpha_{\mathrm{eq}} I_{\mathrm{L}}
$$

obtenu pour

$$
\omega_{\mathrm{S}}-\omega_{\mathrm{L}}=\frac{1}{\tau_{\mathrm{R}}}
$$

avec $\alpha_{\text {eq }}=2 b\left(I_{\mathrm{L}}+I_{\mathrm{S}}\right) \simeq 2 b I_{\mathrm{L}}$ pour les cas de conversion faible qui nous intéressent ici.

$$
\tau_{\mathrm{R}}=\frac{\rho C_{p}}{\lambda_{T}} \frac{1}{\left(k_{\mathrm{L}}+k_{\mathrm{S}}\right)^{2}}
$$

$\beta_{T}$ coefficient de dilatation $\lambda_{T}$ conductivité thermique $C_{p}$ chaleur spécifique à pression constante.

La caractéristique principale de ce nouveau phénomène est sa dépendance en intensité laser. En effet, contrairement à toutes les diffusions stimulées classiques pour lesquelles le gain varie linéairement avec $I_{\mathrm{L}}$, on a ici une variation $I_{\mathrm{L}}^{2}$.

Une conséquence de ceci est que le seuil de puissance laser correspondant à l'apparition de l'onde retour n'est plus indépendant de la focalisation. 
Considérons par exemple un faisceau gaussien

avec

$$
\begin{gathered}
I_{\mathrm{L}}(\rho, z)=\frac{2 P_{\mathrm{L}}}{\pi r^{2}} \mathrm{e}^{-\frac{2 \rho^{2}}{r^{2}}} \\
r=r_{0}\left[1+\left(\frac{z \lambda}{\pi r_{0}^{2} n}\right)^{2}\right]^{1 / 2} .
\end{gathered}
$$

Dans le cas d'une diffusion stimulée classique, l'amplification totale sur l'axe sera :

$$
\log \frac{I_{\mathrm{S}}}{I_{0}}=g \int_{-\infty}^{+\infty} I_{\mathrm{L}}(0, z) \mathrm{d} z=g P_{\mathrm{L}} \frac{2 \pi n}{\lambda}
$$

et dans notre cas

$$
\log \frac{I_{\mathrm{S}}}{I_{0}}=2 \Gamma b \int_{-\infty}^{+\infty} I_{\mathrm{L}}^{2}(0, z) \mathrm{d} z=2 \Gamma b \frac{P_{\mathrm{L}}^{2}}{\pi r_{0}^{2}} \cdot \frac{2 \pi n}{\lambda}
$$

L'apparition d'un signal de retour mesurable correspondant à une amplification déterminée du bruit spontané $I_{0}\left(\log \frac{I_{\mathrm{S}}}{I_{0}} \simeq 30\right)$, le seuil $P_{\mathrm{LS}}$ dans le premier cas sera indépendant de $r_{0}$, dans le deuxième cas proportionnel à $r_{0}$ donc à la focale de la lentille utilisée.

C'est tout d'abord par cette dépendance en focale que nous avons cherché à caractériser le phénomène.

Nous avons utilisé comme onde $I_{\mathrm{L}}$ le deuxième harmonique d'un laser à Rubis déclenché. L'impulsion à $3471 \AA$ avait une durée de 8 ns à mi-hauteur. Le faisceau, monomode longitudinal et spatial à la sortie de l'oscillateur, étant dégradé par la chaîne d'amplification, on peut difficilement parler d'une répartition d'intensité gaussienne. Cependant comme le montre la figure 1 , les résultats précédents restent valables.

Pour tenir compte des aberrations des lentilles de courte focale, nous avons suivi la variation du rapport du seuil d'apparition de l'onde arrière au seuil Bril-

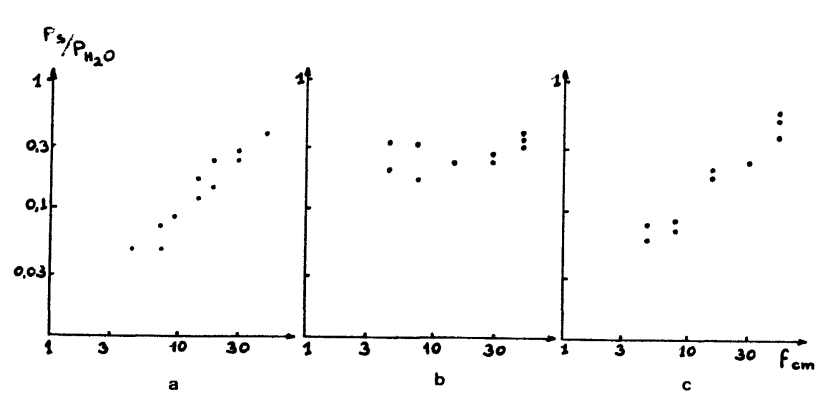

FIG. 1. - Evolution du rapport du seuil d'apparition de l'onde arrière $\boldsymbol{P}_{\mathrm{LS}}$ au seuil Brillouin de l'eau $\boldsymbol{P}_{\mathrm{H}_{2} \mathrm{O}}$ en fonction de la focale. a) benzène ; b) acétonitrile pur; c) acétonitrile + chloronaphtalène.

[Ratio of backward scattering threshold $P_{\mathrm{LS}}$ to threshold $\boldsymbol{P}_{\mathrm{H}_{2} \mathrm{O}}$ for stimulated Brillouin scattering in water versus focal length. $a$ ) benzene; b) pure acetonitrile; $c$ ) acetonitrile $+3 \%$ chloronaphtalene.] louin stimulé de l'eau. Pour les focales supérieures à $10 \mathrm{~cm}$, ce dernier se situe environ à $45 \mathrm{~kW}$.

Le benzène (Fig. 1a) parfaitement transparent à $3471 \AA\left(\alpha<10^{-3} \mathrm{~cm}^{-1}\right)$ montre de façon très claire une dépendance linéaire. Le seul fait de voir apparaître une onde retour, sans changement de fréquence notable, pour des puissances aussi faibles, prouvait d'ailleurs qu'on était en présence d'un nouveau phénomène.

A titre de contre épreuve, dans l'acétonitrile pur (Fig. 1b) transparent également, l'onde renvoyée correspond probablement à la diffusion Brillouin, le rapport des seuils reste constant aux incertitudes de mesure près.

Dans l'idée de voir apparaître une diffusion thermique par absorption à 1 photon, nous avons rendu absorbant l'acétonitrile par addition de $3 \%$ de chloronaphtalène. Malgré un indice d'absorption de $5 \times 10^{-2} \mathrm{~cm}^{-1}$ on peut constater sur la figure $1 c$ que l'absorption à 2 photons domine et donc que $\alpha_{\text {eq }}=2 b I_{L}$ est supérieur à cette valeur.

La dépendance en intensité laser se manifeste aussi sur les caractéristiques spatiales de l'onde rétrodiffusée : la divergence de celle-ci doit être plus grande dans le cas d'une dépendance en $I_{\mathrm{L}}^{2}$ que dans le cas d'une dépendance en $I_{\mathrm{L}}$. Nous avons observé effectivement que le faisceau renvoyé était plus large dans

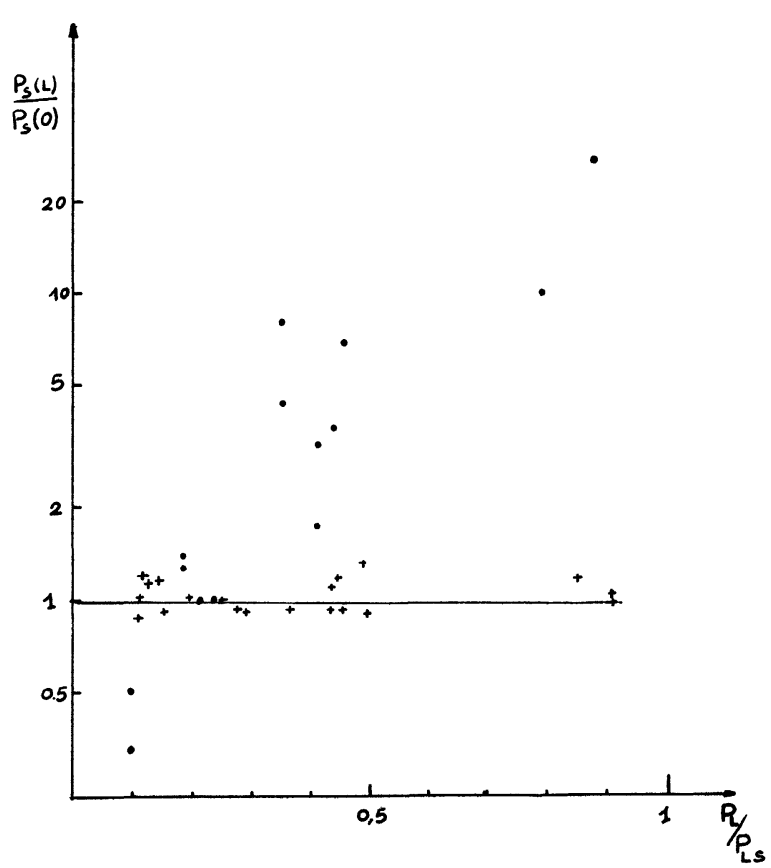

Fig. 2. - Amplification d'une onde $\omega_{\mathrm{s}}$ engendrée dans le benzène ; - mélange acétonitrile + chloronaphtalène ; + acétonitrile pur. Les puissances laser $\boldsymbol{P}_{\mathrm{L}}$ sont ramenées dans les deux cas à la puissance $\boldsymbol{P}_{\mathrm{LS}}$ seuil d'apparition de l'onde rétrodiffusée dans la cuve amplificatrice seule.

[Amplification of a light wave $\omega_{\mathrm{s}}$ generated in benzene. $\bullet$ acetonitrile $+3 \%$ chloronaphtalene $;+$ pure acetonitrile. Laser power $P_{\mathrm{L}}$ is normalized in both cases to the threshold of backward scattering in the amplification cell.] 
le benzène et le mélange acétonitrile + chloronaphtalène que dans l'eau ou l'acétonitrile pur. Dans toutes les expériences décrites ici, les puissances nécessaires à l'apparition des effets de diffusion thermique stimulée sont assez basses pour que l'autofocalisation soit absente.

La signature peu contestable d'une diffusion thermique stimulée est la position en fréquence de l'onde diffusée. En effet, le maximum de gain a lieu pour un écart de fréquence $\omega_{\mathrm{L}}-\omega_{\mathrm{S}}$ très faible, quelques dizaines de $\mathrm{MHz}$, et négatif (diffusion du côté antistokes). Mais une analyse en fréquence aussi fine est relativement délicate $[2,6]$. Nous avons tourné la difficulté par une mesure d'amplification. En effet, les coefficients de diffusion thermique des liquides $\lambda_{T} / \rho C_{p}$ étant assez voisins, l'onde $\omega_{\mathrm{S}}$ créée dans une substance pourra être amplifiée dans une autre.

La figure 2 montre les résultats obtenus pour l'amplification dans le mélange acétonitrile + chloronaphtalène d'une onde engendrée dans une cuve de benzène et l'absence d'amplification de la même onde dans l'acétonitrile pur.

Enfin, pour vérifier si les ordres de grandeurs n'étaient pas déraisonnables, nous avons mesuré directement l'atténuation non linéaire de l'onde $\omega_{\mathrm{S}}$ dans le benzène.

Les mesures de la figure 3 ont été effectuées avec une lentille de $30 \mathrm{~cm}$ de focale et une cuve de $10 \mathrm{~cm}$ de long. Les points marqués + correspondent à des coups de laser pour lesquels la diffusion stimulée est apparue. La fraction d'énergie rétrodiffusée étant faible $\sim 1 \%$, la mesure de transmission n'est pas affectée.

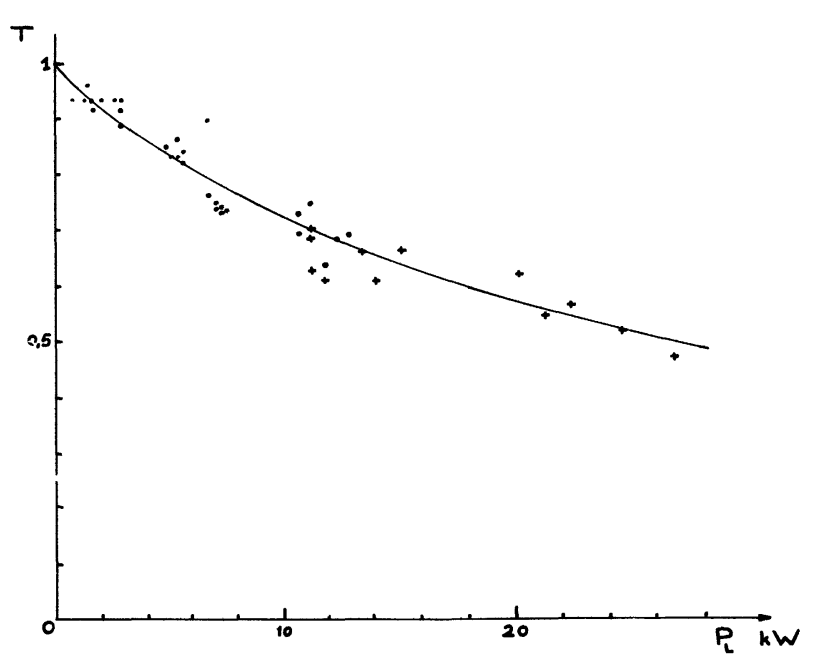

Fig. 3. - Transmission d'une cuve de benzène en fonction de la puissance laser (polarisation linéaire). points sans diffusion stimulée ; + points avec diffusion stimulée. Courbe correspondant

$$
\text { à une loi en } \frac{1}{1+b P_{L} l / s} \text { avec }(b l / s)^{-1}=24 \mathrm{~kW} \text {. }
$$

[Transmission of a benzene cell versus laser power (linear polarization). - without backward scattering; + with backward scattering. Curve drawn follows the law $T=\frac{1}{1+b P_{\mathrm{L}} l / s}$ with

$$
(b l / s)^{-1}=24 \mathrm{~kW} \text {.] }
$$

Le faisceau utilisé étant comme nous l'avons signalé assez médiocre, nous nous sommes contentés d'une approximation simple pour l'analyse des mesures : faisceau de section constante $s$ sur une longueur $l$.

La transmission dans ce cas est de la forme

$$
T=\frac{1}{1+b I l}=\frac{1}{1+b P l / s} \text {. }
$$

La courbe continue de la figure 2 correspond à une telle loi avec $(b l / s)^{-1}=24 \mathrm{~kW}$.

Une évaluation du rapport $l / s$ peut être déduite du seuil Brillouin de l'eau. La référence [4] donne une valeur de l'indice de gain $g_{\mathrm{B}}=6,6 \times 10^{-3} \mathrm{~cm} / \mathrm{MW}$ à $6943 \AA$.

Dans l'expression donnant $g_{\mathrm{B}}$ :

$$
g_{\mathrm{B}}=\frac{\omega_{\mathrm{S}}^{2} \gamma_{\mathrm{e}}^{2}}{c^{3} n v \rho_{0} \Gamma_{\mathrm{B}}}
$$

La largeur de raie Brillouin $\Gamma_{\mathrm{B}}$ varie comme $\omega_{\mathrm{S}}^{2}$ en l'absence de dispersion hypersonique [7]. Le seul terme dépendant de la fréquence est donc

$$
\gamma_{\mathrm{e}}=\rho\left(\frac{\partial \varepsilon}{\partial \rho}\right)_{T}=\frac{\left(n^{2}-1\right)\left(n^{2}+2\right)}{3}
$$

dont la variation est de l'ordre de $10 \%$.

Avec une valeur $g_{\mathrm{B}}$ de $8 \times 10^{-3} \mathrm{~cm} / \mathrm{MW}$ à $3471 \AA$ et une amplification au seuil

$$
\frac{I_{\mathrm{S}}}{I_{0}}=\exp \left\{g_{\mathrm{B}} P_{\mathrm{L}} l / s\right\}=e^{30} .
$$

On obtient un rapport $l / s$ de $8 \times 10^{4} \mathrm{~cm}^{-1}$.

Pour une onde gaussienne, d'après la relation (4) la quantité équivalente serait $2 \pi n / \lambda=23 \times 10^{4} \mathrm{~cm}^{-1}$.

En suivant l'évolution de la transmission du benzène en fonction de la position de la cuve par rapport à la lentille, nous avons estimé que la longueur $l$ était de l'ordre de $5 \mathrm{~cm}$.

L'indice d'atténuation équivalent $\alpha_{\text {eq }}$ est donc de $0,2 \mathrm{~cm}^{-1}$ pour une intensité $I_{\mathrm{L}}$ de $200 \mathrm{MW} / \mathrm{cm}^{2}$.

En utilisant l'expression (3) pour l'indice de gain thermique stimulé, nous pouvons encore transposer la valeur numérique $g_{\mathrm{B}} / \alpha=0,57 \mathrm{~cm}^{2} / \mathrm{MW}$ à $6943 \AA$ (Réf. [4]) à la longueur d'onde moitié :

$$
g_{\mathrm{B}} / \alpha=0,35 \mathrm{~cm}^{2} / \mathrm{MW} \text {. }
$$

Avec cette valeur, on obtiendrait une amplification en régime permanent

$$
\frac{I_{\mathrm{S}}}{I_{0}}=\exp \left[\frac{g_{\mathrm{B}}}{\alpha} \cdot \alpha_{\mathrm{eq}} \cdot P_{\mathrm{L}} \cdot l / s\right]=e^{60}
$$

au seuil d'apparition de l'onde retour.

Cette valeur peut sembler un peu grande mais il faut rappeler que nous faisons cette estimation dans l'hypothèse d'un régime permanent, compte tenu de la valeur respective de la durée d'impulsion laser (8 ns) et du temps de relaxation des ondes thermiques $\tau_{R}$ 
(3,2 ns pour $\lambda=3471 \AA$ ) ; cette hypothèse n'est pas justifiée, ce qui nous conduit à surestimer le gain [8].

D'autre part, nous avons admis que l'énergie prise aux ondes $\omega_{\mathrm{L}}$ et $\omega_{\mathrm{S}}$ était intégralement transformée en chaleur dans un temps court durant $\tau_{R}$ ce qui n'est peut-être pas le cas. Ces mesures nous permettent d'évaluer la section efficace d'absorption à deux photons du benzène

$$
\sigma \simeq 4 \times 10^{-50} \mathrm{~cm}^{4} . \mathrm{s}
$$

pour un niveau d'énergie de $7,15 \mathrm{eV}$.

Cette valeur est en bon accord avec la fourchette de 2 à $100 \times 10^{-50} \mathrm{~cm}^{4}$. s calculée dans la référence [9] pour la transition ${ }^{1} \mathrm{E}_{2 \mathrm{~g}} \leftarrow{ }^{1} \mathrm{~A}_{1 \mathrm{~g}}$ à $7,7 \mathrm{eV}$.

La variation du seuil et la diminution de l'absorption lorsqu'on introduit une lame quart d'onde sur le faisceau indique que cette valeur est divisée par deux pour une onde $\omega_{\mathrm{L}}$ polarisée circulairement.

La valeur de $g_{\mathrm{B}} / \alpha$ pour l'acétonitrile est sans doute assez proche de celle du benzène. Compte tenu de la faible concentration dans notre mélange, la section efficace à deux photons du chloronaphtalène doit être très grande : $\sigma \simeq 10^{-48} \mathrm{~cm}^{4}$.s.

A cause des fluctuations inhérentes à tout phénomène de diffusion stimulée, nous ne pensons pas que cette méthode puisse servir à mesurer avec précision des sections efficaces d'absorption à deux photons. La mesure par défocalisation thermique par exem- ple [10] est certainement d'une sensibilité et d'une précision meilleures. Cependant nous pensons qu'elle offre un certain intérêt :

Par sa simplicité de mise en œuvre, elle peut permettre de se faire rapidement une idée sur les ordres de grandeur (comme par exemple pour le chloronaphtalène dans cette étude).

Elle donne aussi une indication sur le temps de relaxation thermique $\tau$ du niveau excité à deux photons : l'amplitude de la défocalisation thermique est reliée de façon simple au seuil stimulé pour un liquide donné tạt que $\tau<\tau_{R}$, un comportement différent signalerait une relaxation thermique longue.

Enfin il ne faut pas oublier que ce phénomène peut apparaître comme élément perturbateur dans les mesures, les puissances renvoyées allant jusqu'à $20 \%$ de la puissance incidente lorsqu'on est largement au-dessus du seuil $\left(P_{\mathrm{L}} \simeq 10 P_{\mathrm{LS}}\right)$.

Par ailleurs, cette étude expérimentale s'est limitée au cas dégénéré d'absorption de deux photons identiques. Il n'y a pas d'interdiction de principe à ce que deux ondes différentes $\omega_{1}$ et $\omega_{2}$ dont les directions de propagation font entre elles un angle quelconque se réfléchissent mutuellement avec un gain proportionnel à $I_{1} I_{2}$. Le seul problème est de trouver un matériau pour lequel la section efficace à $\omega_{1}+\omega_{2}$ soit plus grande qu'à $2 \omega_{1}$ ou $2 \omega_{2}$.

\section{Bibliographie}

[1] Herman, R. M., Gray, M. A., Phys. Rev. Lett. 19 (1967) 824.

[2] Rank, D. H., Cho, C. W., Foltz, N. D. et Wiggins, T. A., Phys. Rev. Lett. 19 (1967) 828.

[3] GrRes, F., C.R. Acad. Sci. 266 (1968) 596.

[4] KAISER, W. et MAIER, M., Lasers Handbook (North Holland Publishing Company Amsterdam), 1972, p. 1077

[5] GrRes, F., MAYER, G., C.R. Acad. Sci. 266 (1968) 8.

[6] GrRes, F., Thèse, Paris 1968.
[7] Chino, R. Y., Fleury, P. A., Physics of Quantum Electronics (Mc Graw Hill), 1966, p. 241.

[8] Rother, W., MeYer, H. et KaISER, W., Z. Naturforsch 25a (1970) 1136.

[9] Honig, B., Jortner, J. et SzÖKe, A., J. Chem. Phys. 46 (1967) 2714.

[10] Twarowski, A. S. et Kliger, D. S., Chem. Phys. 20 (1977) 259. 